\title{
Zur Schraubentheorie von Sir Robert Ball.*)
}

\author{
Von
}

F. KLEWN in Göttingen.

Sir Robert Ball hat seine langjährigen Untersuehungen über Sehraubentheorie im vorigen Jahre in einem stattlichen Bande zusammengefaßt**), der nicht verfehlen kann, dieser geometrischen Weiterbildung der Mechanik starrer Körper erneat das allgemeine Interesse zuzuwenden. $Z_{\text {wei }}$ Vorzüge sind es insbesondere, die dem Ballschen Werke von vornherein einen zahlreichen Leserkreis sichern dürften, nämlich die Anschaulichleit und der elementare Charakter seiner grundlegenden Fntwicklungen. Ich wünsche diese Vorzüge lebhaft anzuerkennen, will aber andererseits hervorheben, daß dieselben von einem gewissen Verzicht auf die Darlegung der im weiteren Verfolg der Theorie notwendig in Betracht kommenden tiefer greifenden Fragen begleitet werden (wie dies übrigens der Verfasser selbst an verschiedenen Stellen seines Buches deutlich hervorhebt).**)

Jedenfalls möchte ich im folgenden eìnige Ergänzangen zum Ballschen Werke geben, die manchem Leser willkommen sein dürften. Diese Ergänzungen betreffen erstlich die allgemeine Systematik des Gebietes im Sinne moderner invariantentheoretischer (oder gruppentheoretischer) Prinzipien, zweitens aber die Verwendung der Schraubentheorie in der Lehre

7) Abgedruckt aus dem $47^{\text {ten }}$ Bande der Zeitschrift für Mathematik und Physik (1902); am SchluB sind einige ,nachträgliche Bemerkungen" zugefügt.

**) A Treatise on the Theory of Serews, Cambridge 1900.

**) Man vergl. z. B. die amüsante Auseinandersetzung, die der Verf. 1887 über die Ziele seiner Untersuchungen vor der British Association in Manchester gab und die nun aus den bez. Reports auf pg. 496-509 des vorliegenden Buches wieder abgedruckt ist. Eine Kommission ist niedergesetzt, um die Bewegungen eines starren Körpers zu untersuchen. "Let it suffice for us", sagt der Präsident der Kommission gleich zu Anfang, "to experiment upon the dynamies of this body so long it remains in or near the position it now occupies. We may leave to some more ambitions committee the task of following the body in all conceivable gyrations through the universe." 
von den enalichen Bewegangen starrer Körper (wo ich übrigens in der Hauptsache nur systematisch zusammenstelle, was zerstreut in der Literatur vorliegt). Ich darf vielleicht hinzufügen, daB ich die betreffenden Überlegungen seit Jahren in Vorlesungen und gelegentlichen Vorträgen wiederholt zur Geltung gebracht habe; speziell knüpfe ich mit den Darlegungen der nächstfolgenden Paragraphen an meine eigenen Beiträge zur Liniengeometrie und Schraubentheorie aus den Jahren 1869 und 1871*), sowie an die Auseinandersetzung meines Erlanger Programmes von 1872\%\%) an. Es hat seinen guten Sinn, daB ich mich dabei von vornherein der Methoden der analytischen Geometrie bediene; in der Tat meine ich, dadurch die in Betracht kommenden Beziehungen kürzer und präziser bezeichnen zu können, als dies auf andere Weise möglich wäre.

\section{$\S 1$.}

\section{Von der rationellen Klassifikation geometrischer und mechanischer Größen.}

Als Hauplgruppe räumlicher Änderungen bezeichne ich in meinem Erlanger Programme den Inbegriff der Bewegungen des Raumes und seiner Änlichkeitstransformationen. Es möge ein rechtwinkliges Koordinatensystem zugrunde gelegt werden; ich deute an, wie die Operationen der Hauptgruppe auf die zugehörigen Punktkoordinaten wirken. Wir haben erstlich für Drehungen um den Anfangspunkt Formeln folgender Bauart:

$$
\left\{\begin{array}{l}
x_{1}=a x+b y+c z \\
y_{1}=a^{\prime} x+b^{\prime} y+c^{\prime} z \\
z_{1}=a^{\prime \prime} x+b^{\prime \prime} y+c^{\prime \prime} z
\end{array}\right.
$$

dabei hat man zwischen den $a, b, c, \cdots$ die bekannten Relationen und insbesondere ist jede dieser Größen gleich der ihr in der Determinante

$$
\left|\begin{array}{lll}
a & b & c \\
a^{\prime} & b^{\prime} & c^{\prime} \\
a^{\prime \prime} & b^{\prime \prime} & c^{\prime \prime}
\end{array}\right|
$$

zugehörigen Unterdeterminante. Wir haben ferner für Parallelverschiebungen des Raumes Formeln, die ich so bezeichne:

$$
x_{1}=x+A, \quad y_{1}=y+B, \quad z_{1}=z+C,
$$

*) Math. Annalen, Bd. 2 und 4. Vgl. insbesondere die "Notiz, betreffend den Zusammenhang der Liniengeometrie mit der Mechanik starrer Körper" in Bd. 4 daselbst, pg. $403-415$ (1871).

*) „Vergleichende Betrachtungen über nenere geometrische Forschungen“ (Erlangen 1872), abgedruckt in Bd. 43 der Math. Annalen and anderwärts. 
endlich für diejenigen Ähnlichkeitstransformationen, die den Koordinatenanfangspunkt festlassen:

$$
x_{1}=\lambda x, \quad y_{1}=\lambda y, \quad z_{1}=\lambda z
$$

unter ihnen mögen wir die Inversionen

$$
x_{1}=-x, \quad y_{1}=-y, \quad z_{1}=-z
$$

besonders hervorheben. Die Formeln für beliebige Transformationen der Hauptgruppe ergeben sich aus (1), (2), (3) durch Kombination; wir mögen dementsprechend die (1), (2), (3) als erzeugende Substitutionen der Hauptgruppe bezeichnen. Es handelt sich dabei zunächst um Raumtransformationen bei festem Koordinatensystem. Es steht aber nichts im Wege, die Formeln auch so zu interpretieren, dab sie bei festgehaltenem Raume den Übergang je zu einem neuen rechtwinkligen Koordinatensysteme vorstellen (so daB es sich bei den Operationen der Hauptgruppe überhaupt am die allgemeinste Transformation der rechtwinkligen Koordinaten handelt). Wir werden in der Folge diese Auffassung, die zumal bei den Verallgemeinerungen eine Kleinigkeit bequemer scheint, bevorzugen. Die Formeln (1) und (2) ergeben dann zusammengenommen die allgemeinste Abänderung des rechtwinkligen Koordinatensystems durch Bewegung, Formel (4) den Übergang zu einem inversen Koordinatensystem, Formel (3) für die allein nur noch in Betracht kommenden positiven Werte von $\lambda$ die allgemeinste Abänderung, welche aus geänderter Wahl der Längeneinheit resultiert.

Wir legen nunmehr nicht bloB Punkte, sondern beliebige andere geometrische Gebilde hinsichtlich unseres Koordinatensystems durch „Koordinaten" fest, wobei wir uns diese Gebilde in geeigneter Weise durch Punkte definiert denken, so daB ihre "Koordinaten" Verbindungen verschiedener Reihen von Punktkoordinaten sind. Den Inbegriff der solcherweise zur Festlegung eines geometrischen Gebildes dienenden Koordinaten mögen wir jeweils als ,geometrische Größs" bezeichnen. Und nun ruht die rationelle Klassifikation geometrischer Größen, von der im folgenden ausgegangen werden soll, einfach darauf, daB wir zusehen, wie sich die in Betracht kommenden Koordinaten bei den Operationen (1), (2), (3) bez. (4) (und also überhaupt bei den Operationen der Hauptgruppe) verhalten. Wir werden alle diejenigen und nur diejenigen geometrischen Größen als gleichartig ansehen, deren Koordinaten bei den Operationen der Hauptgruppe die gleichen Äderungen erleiden. Erleiden aber die Koordinaten zweier Gebilde verschiedene Änderungen, so ergibt sich die geometrische Beziehung der beiden Arten geometrischer Größen zueinander unmittelbar und in erschöpfender Weise durch den, Vergleich der beiderlei Änderungen. 
Ansführungen zu diesem Prinzip enthält $u$. a. der neuerdings erschienene Artikel von Abraham über die geometrischen Grundbegriffe in der Mechanik der deformierbaren Körper, Bd. IV der mathematischen Encyklopädie, Art. 14. In der Sache hat man selbstverständlich immer dem Prinzip entsprechend verfahren. Insbesondere ist die in der Mechanik (nnd Physik) übliche Unterscheidung der geometrischen Größen nach ihrer Dimension nichts anderes als eine Inbetrachtnahme der Substitutionen (3) im Sinne unseres Prinzips (wobei man sich stillschweigend auf positive Werte von $\lambda$ beschränkt). In dieser Bemerkung liegt zugleich, wie unser Prinzip auf allgemeine, mechanische oder physikalische Größen auszudehnen ist. Es ist weiterhin bequem neben der Längeneinheit und Zeiteirheit nicht, wie sonst üblich, eine Masseneinheit, sondern eine Krafteinheit eingeführt zu denken. Man wird daraufhin den Formeln (3) noch diejenigen zur Seite stellen, die sich auf die Änderung der Zeiteinheit, bez. die Anderung der Krafteinheit beziehen:

$$
t_{1}=\varrho t
$$

$$
P_{1}=\sigma P
$$

man wird dann sagen, daB die Formeln (1)-(6) zusammen die Hauptgruppe der Mechanik (bez. der Physili) definieren, und ferner die mechanischen (bez, die physikalischen) Größen nach dem Verhalten einteilen, welches ihre Koordinaten bei den Operationen dieser Hauptgruppe zeigen. Übrigens werden wir auf diese erweiterten Festsetzungen nur bei Gelegenheit zurückkommen; für die laufenden Entwicklungen genügt uns die Inbetrachtnahme der räumlichen Hauptgruppe.

\section{$\S 2$.}

\section{Koordinaten für die unendlich kleine Bewegung eines starren} Körpers, sowie für die an ihm angreifenden Kraftsysteme.

Eine unendlich kleine Bewegung mag durch folgende Formeln vorgestellt sein:

$$
\left\{\begin{array}{l}
d x=(-r y+q z+u) d t \\
d y=(-p z+r x+v) d t \\
d z=(-q x+p y+w) d t
\end{array}\right.
$$

Wir bezeichnen die Größen

$$
p, q, r, u, v, w
$$

als die Koordinaten der instantanen Geschwindigkeit, dagegen die Größen

$$
p d t, q d t, v d t, u d t, v d t, w d t
$$

als die Koordinaten der unendlich kleinen Bewegung selbst. 
Kräfte am starren Körper stellen wir in üblicher Weise durch Strecken dar, welche auf bestimmte gerade Linien aufgetragen und längs dieser geraden Linien verschiebbar sind. Dabei werden wir die Länge dieser Strecken je der Größe der Kräfte gleich setzen; es ist gleichgültig, ob wir uns dabei die Kräfte sämtlich als StoBkräfte oder als kontinuierlich wirkende Kräfte denken.*) Es seien $x, y, z$ bez. $x^{\prime}, y^{\prime}, z^{\prime}$ Anfangsund Endpunkt einer , linienfiüchtigen" Strecke. Dam hat man in üblicher Weise als Koordinaten derselben:

$$
x^{\prime}-x, \quad y^{\prime}-y, \quad z^{\prime}-z, \quad y z^{\prime}-y^{\prime} z, \quad z x^{\prime}-z^{\prime} x, \quad x y^{\prime}-x^{\prime} y
$$

dieselben sechs Größen werden als Koordinaten der Kraft gelten, sofern man die Länge $l$ der Strecke gleich der Zahl $P$ gewählt hat, welche die Gröbe der Kraft miBt. Wollen wir die Abhängigkeit von der Wahl der Krafteinheit und der Längeneinheit deutlicher hervorkehren, so wird es zweckmäßiger sein, als Koordinaten der Kraft folgende sechs Größen zu bezeichnen:

$\frac{P}{l}\left(x^{\prime}-x\right), \frac{P}{l}\left(y^{\prime}-y\right), \frac{P}{l}\left(z^{\prime}-z\right), \frac{P}{l}\left(y z^{\prime}-y^{\prime} z\right), \frac{P}{l}\left(z x^{\prime}-z^{\prime} x\right), \frac{P}{l}\left(x y^{\prime}-x^{\prime} y\right)$.

Als Kräftesystem bezeichnen wir den Inbegriff beliebig vieler auf den starren Körper wirkender Einzelkräfte und wählen als Koordinaten desselben die Summen der zusammengehörigen Koordinaten dieser Einzelkräfte. Solcherweise erhalten wir als Koordinaten eines Kräftesystems die sechs Größen:

$$
\begin{array}{lll}
X=\sum \frac{P_{i}}{l_{i}}\left(x_{i}^{\prime}-x_{i}\right), & Y=\sum \frac{P_{i}}{l_{i}}\left(y_{i}^{\prime}-y_{i}\right), & Z=\sum \frac{P_{i}}{l_{i}}\left(z_{i}^{\prime}-z_{i}\right), \\
L=\sum \sum \frac{P_{i}}{l_{i}}\left(y_{i} z_{i}^{\prime}-y_{i}^{\prime} z_{i}\right), & M=\sum \frac{P_{i}}{l_{i}}\left(z_{i}^{\prime} x_{i}^{\prime}-z_{i}^{\prime} x_{i}\right), \quad N=\sum \frac{P_{i}}{l_{i}}\left(x_{i} y_{i}^{\prime}-x_{i}^{\prime} y_{i}\right) .
\end{array}
$$

Es wird nunmehr darauf ankommen, zuzusehen, wie sich die Koordinaten $p, q, r, u, v, w(8)$ und die jetzt eingeführten $X, Y, Z, L, M, N$ bei den Operationen (1)-(6) der Hauptgruppe verhalten. Ich stelle hier die Resultate einfach zusammen:

1. Drehung un den Koordinatenanfangspunkt (Formel (1)).

Die Koordinaten $p, q, r$ und die $u, v, w$, andererseits die $X, Y, Z$ and die $L, M, N$ erleiden je für sich genau dieselbe Substitation wie die Punktkoordinaten $x, y, z$. (Dies Resultat ruht wesentlich auf dem oben herrorgehobenen Umstande, dab die Substitutionskoeffizienten $a, b, c, \cdots$ ihren bez. Unterdeterminanten gleich sind.)

*) Die Unterscheidung tritt erst ein, wenn wir sur Kinetik schreiten, wo dann die Verabredung sein wird, daB die Einheit der Stokkraft an irgend oinem Massenpunkte instantan dieselbe Geschwindigkeitsänderung hervorbringt, wie die Einheit der kontinuierlichen Kraft während der Zeiteinheit. 


\section{Verschiebung (Formel (2)).}

Die $p, q, r$, andererseits die $X, Y, Z$ bleiben ungeändert. Dagegen erleiden die $u, v, w$ die folgende Substitution:

$$
\left\{\begin{array}{l}
u_{1}=u-C q+B r \\
v_{1}=v-A r+C p \\
w_{1}=w-B p+A q
\end{array}\right.
$$

und genau entsprechende Formeln ergeben sich für $L, M, N$ :

$$
\left\{\begin{array}{l}
L_{1}=L-C Y+B Z, \\
M_{1}=M-A Z+C X, \\
N_{1}=N-B X+A Y .
\end{array}\right.
$$

3. Ahnlichkeitstransformation (Formel (3), bez. (4)).

lst $\lambda$ positiv, so werden

$$
p_{1}, q_{1}, r_{1}, u_{1}, v_{1}, w_{1} \text { bez. gleich } p, q, r, \lambda u, \lambda v, \lambda w
$$

und genau so

(12) $X_{1}, Y_{1}, Z_{1}, L_{1}, M_{1}, N_{1}$ bez. gleich $X, Y, Z, \lambda L, \lambda M, \lambda N$.

Dagegen tritt bei negativem $\lambda$ ein Unterschied ein, der sich am einfachsten darin ausprägt, daß bei Inversion

$$
p_{1}, q_{1}, r_{1}, u_{1}, v_{1}, w_{1} \text { gleich } p, q, r,-u,-v,-w,
$$

dagegen

(13') $X_{1}, Y_{1}, Z_{1}, L_{1}, M_{1}, N_{1}$ gleich $-X,-Y,-Z, L, M, N$

werden. (Dieser Unterschied kommt dadurch hervor, dab die in den Formeln (10) auftretenden Längen $l_{i}$ absolute Beträge sind, welche als solche ihr Vorzeichen bei Inversion nicht wechseln.)

4. Anderung der Zeiteinheit (Formel (5)).

(14) $p_{1}, q_{1}, r_{1}, u_{1}, v_{1}, w_{1}$ sind bez. gleich $\frac{\rho}{\varrho}, \frac{q}{\varrho}, \frac{r}{\varrho}, \frac{u}{\rho}, \frac{v}{\varrho}, \frac{w}{\varrho} ;$

die Koordinaten des Kräftesystems bleiben ungeändert.

5. Anderung der Krafteinheit (Formel (6)).

Die $p, q, r, u, v, w$ bleiben ungeändert, dagegen werden

(15) $X_{1}, Y_{1}, Z_{1}, L_{1}, M_{1}, N_{1}$ bez. gleich $\sigma X, \sigma Y, \sigma Z, \sigma L, \sigma M, \sigma N$.

Indem wir uns der Kürze halber auf die Hauptgruppe räumlicher Anderungen beschränken, werden wir zusammenfassend sagen können:

Bei bloßer Bewegung des Koordinatensystems, ebenso auch bei Ähntichkeitstransformation von positivem Ähnlichkeitsmadul, transformieren sich die Krafthoordinaten

$$
X, \quad Y, \quad Z, \quad L, \quad M, \quad N
$$


genau wie die Geschwindigheitskoordinaten

$$
p, q, \quad r, u, v, w .
$$

Dagegen tritt bei Inversion des Koordinatensystems ein abweichendes Verhalten ein; während die

$$
p, q, r, u, v, w \text { in } p, q, r,-u,-v,-w
$$

ïbergehen, verwandeln sich die

$$
\mathrm{X}, Y, Z, L, M, N \text { bez. in }-\mathrm{X},-Y,-Z, L, M, N \text {. }
$$

$\S 3$.

Die Analogie der unendlich kleinen Bewegungen und der Kräftesysteme (beim starren Körper). Schraubengrößen der ersten und zweiten Art. Ballsche Sehranben.

Durch die Formeln des vorigen Paragraphen ist die Analogie von unendlich kleinen Bewegungen und Kräftesystemen, welche die ganze Mechanik der starren Körper und insbesondere die Ballsche Schraubentheorie durchzieht, auf das. klarste begründet und gleichzeitig umgrenat.

Bemerken wir vorab, daß das GröBensystem

$$
p d t, \quad q d t, \quad r d t, \quad u d t, \quad v d t, \quad w d t
$$

vermöge der Formeln (7) ohne weiteres eine (unendlich kleine) Schraubung des Raumes der $x, y, z$ (von bestimmter Achse, Ganghöhe und Amplitude) bedeutet, das GröBensystem der

$$
p, q, r, u, v, w
$$

dementsprechend eine Schraubungsgeschwindigkeit. Ich will in diesem Sinne den Inbegriff der $p, q, r, u, v, w$ fortan als eine Schraubengröße bezeichnen, genauer, wenn es darauf ankommt, als eine Schraubengröße erster Art.

Nunmehr wolle man den Inbegriff der Koordinaten eines Kräftesystems, also die in (10) definierten

$$
X, \quad Y, Z, L, \quad M, N
$$

zum Vergleich heranziehen. Wir wollen insbesondere ein Kräftesystem und eine Schraubengröße erster Art in Zusammenhang bringen, indem wir setzen:

$$
X=p, \quad Y=q, \quad Z=r, \quad L=u, \quad M=v, \quad N=w,
$$

und uns fragen, wie weit diese Zusammenordnung eine vom Koordinatensystem unabhängige Bedeutung hat (also gegenüber den Operationen der 
Haaptgruppe invariant ist). Zunächst ergeben die Formeln $(14$, (15) des vorigen Paragraphen, daß die Zuordnung von der Wahl der Zeiteinheit und der Krafteinheit abhängig ist. Ferner aber ergeben die Formeln für Drehung, Parallelverschiebung und Ähnlichkeitstransformation mit positivem Ähnlicikeitsmodul, daß die Zuordnung von allen in diese Worte einbegriffenen Änderungen des räumlichen Koordinatensystems unabhängig ist. Endlich die Formeln (13), (13'), daß sich die Zuordnung bei Inversion in ihr Gegenteil verkehrt:

(17) $X_{1}=-p_{1}, Y_{1}=-q_{1}, Z_{1}=-r_{1}, L_{1}=-u_{1}, M_{1}=-v_{1}, N_{1}=-w_{1}$.

Die geometrische Überlegung bestätigt das so formulierte Resultat. natürlich Schritt für Schritt. Ich will, um dies im Detail auszuführen, angeben, dab die Achse der Schraubengeschwindigkeit $p, q, r, u, v, w$ die Linienkoordinaten hat:

$$
p: q: r: u-k p: v-k q: w-k r,
$$

wo der "Parameter"

$$
k=\frac{p u+q v+r w}{p^{2}+q^{2}+r^{2}}
$$

und daB die Drehgeschwindigkeit um diese Achse die Komponenten $p, q, r$, die Translationsgeschwindigkeit längs der Achse die Komponenten $k p, k q, k r$ besitzt. Genau entsprechend kann man bei einem Kräftesystem $X, Y, Z$, $L, M, N$ eine Zentralachse finden, deren Linienkoordinaten durch

$$
X: Y: Z: L-k X: M-k Y: N-k Z
$$

gegeben sind, unter $k$ die Größe

$$
k=\frac{X L+Y M+Z N}{X^{2}+Y^{2}+Z^{2}}
$$

verstanden, und das Kräftesystem läßt sich dann auf eine Einzelkraft mit den Komponenten $X, Y, Z$ entlang dieser Achse und ein Paar mit den Komponenten $k X, k Y, k Z$ in einer zur Achse senkrechten Ebene reduzieren. Die Zusammenordnung verlangt, der Drehgeschwindigkeit um die Achse die längs der Achse wirkende Einzelkraft und der in Richtung der Achse liegenden Translationsgeschwindigkeit ein Paar in einer zur Achse senkrechten Ebene glẹich zu setzen. Hierzu ist selbstverständlich eine vorherige Verständigung über die Zeiteinheit und die Krafteinheit notwendig. Erst wenn dies geschehen, kann man sagen, daß die Intensität eines Kräftesystems (gemessen durch $\sqrt{X^{2}+Y^{2}+Z^{2}}$ ) gleich der durch $\sqrt{p^{2}+q^{2}+r^{2}}$ gemessenen Intensität einer Geschwindigkeit sei. Darïber hinaus aber brauchen wir eine Verabredung, welchen Sinn um die Achse man einem entlang der Achse weisenden Sinne zuweisen will; - ob den- 
jenigen Sinn um die Achse, der beim Entlangblicken längs der Achse in der vorgegebenen Richtung durch die Bewegung des Uhrzeigers gegeben ist, oder den entgegengesetzten. Erst durch diese Verabredung wird die Zusammenordnung von Kräftesystem und Geschwindigkeit eindeutig. Jede solche Verabredung verwandelt sich aber bei Inversion der Figur bekanntlich in ihr Gegenteil, und dies ist, was dureh Formel (17) ausgedrückt wird.

Der Inbegriff der ( $X Y Z L M N)$ steht also zwar dem Inbegriff der (pqruvw), d. h. der SchraubengröBe erster Art sehr nahe, ist aber nicht selbst eine SchraubengröBe erster Art. Wir werden ibn als Schraubengröße zweiter Art bezeichnen. Die Zusammenordnung der beiderlei Größenarten aber werden wir so in Worte fassen, daß wir sagen:

Nachdem Zeiteinheit und Krafteinheit festgelegt sind, gehören zu einer Schraubengröße zweiter Art immer noch zwei (entgegengesetzt gleiche) Schraubengrößen erster Art, und umgekehrt; die Zusammengehörigkeit wird erst eine eindeutige, wenn man im angegebenen Sinne eine Verabredung über rechts und links hinzufügt.

Neben die so besprochenen SchraubengröBen erster und zweiter Art treten dann drittens als engverwandte geometrische Gebilde die Ballschen Sckrauben selbst. Die Ballsche Schraube ist der Inbegriff der um eine Achse hernmgelegten Schraubenlinien von gegebenem Windungssinn, die eine bestimmte Ganghöhe haben, oder, wie Ball sagt, der Inbegriff von Zentralachse und Parameter (pitch). Die so definierte Ballsche Schraube ist mit dem Nullsystem, das jedem Punkte die Normalebene der durch ihn gehenden Schraubenlinien zuordnet, oder auch mit dem linearen Linienkomplex, der von den Normalen sämtlicher Schraubenlinien gebildet wird, eineindentig zusammengeordnet; ob ich von der Ballschen Schraube, dem Nullsystem oder dem linearen Komplex spreche, ist für den hier vertretenen Standpunkt dasselbe. Jedes dieser Gebilde wird durch die Verhältnisse $X: Y: Z: L: M: N$ der Koordinaten einer Schraubengröbe zweiter Art, oder auch durch die Verhältnisse $p: q: r: u: v: w$ der Koordinaten oder SchraubengröBe erster Art festgelegt. In der Tat verschwindet, wenn man sich auf die Betrachtung dieser „Verhältnisse" beschränkt, der Unterschied der bęiden Arten von SchraubengröBen. Entsprechend gibt es nur eine Art Ballscher Schrauben. Zu jeder Ballschen Schraube gehören unendlich viele Schranbengrößen erster wie zweiter Art, die sich untereinander durch Intensität und Sinn unterscheiden.

Hiermit dürfte der Zusammenhang der verschiedenen in Betracht kommenden Gebilde so vollständig dargelegt sein, als man wünschen mag. Die einzelne "Schraube" ist Trägerin von unendlich vielen .,Schraubengrößen erster and zweiter Art". Indem wir die letzteren sprachlich nnterscheiden, dürfte zugleich dem immer wiederkehrenden MiBrerständnisse, 
als handele es sich bei der Zusammenordnung der zweierlei Schraubengrößen um einen kausalen Zusammenhang, nach Möglichkeit vorgebeugt sein.*)

\section{$\S 4$.}

\section{Über die Invarianten der Schraubengrößen und die Begründung der Artunterscheidung aus dem Arbeitsbegriff.}

Die gegenseitige Beziehung der beiden Arten von Schraubengrößen findet einen sehr prägnanten Ausdruck, wenn man ihre Invarianten betrachtet, d. h. diejenigen aus ihren Koordinaten gebildeten rationalen ganzen Funktionen, welche gegenüber den Operationen der Hauptgruppe entweder überhaupt ungeändert bleiben oder sich nur um einen Faktor ändern. Ich werde mich hier der Kürze wegen auf diejenigen Operationen der Hauptgruppe beschränken, die entweder Bewegungen vorstellen oder aus Bewegungen durch Hinzutreten einer Inversion entstehen, und die ich mit Herrn Study als Umlegungen bezeichnen will.

Als Invarianten der einzelnen Schraubengröße ergeben sich bekanntlich erstens die Ausdrücke:

$$
p^{2}+q^{2}+r^{2} \text { bez } X^{2}+Y^{2}+Z^{2},
$$

die bei Bewegungen und Umlegungen gleichmäBig ungeändert bleiben, zweitens aber die folgenden:

$$
p u+q v+r w \text { bez. } X L+Y M+Z N
$$

dieselben bleiben bei beliebigen Bewegungen ungeändert, kehren aber bei Umlegungen (wie aus ibrem Verhalten bei Inversion hervorgeht) ihr Zeichen um. Wir werden dementsprechend die (20) als gerade Invarianten bezeichnen, die (21) als schiefe, oder auch die (20) als Skalare der ersten Art, die (21) als Skalare der zweiten Art.*)

Die hiermit eingeführte Unterscheidung überträgt sich selbstverständlich auf diejenigen „simultanen" Invarianten zweier Schraubengrößen der-

*) Vergl. die Erörterungen in meiner oben genannten Notiz, Math. Ann. Bd. 4, pg. 403 ff. Die Hartnäckigkeit des Mißverständnisses hat offenbar eine psychologische Wurzel. Wir sind durch unsere tägliche Beschäftigung gewöhnt, wenn wir eine Einzelkraft auf einen Körper wirken lassen, diese auf den Schwerpunkt des Körpers zu richten, worauf sie natürlich Translation des Körpers erzeugt. Von hier aus hat sich zwischen den beiden Dingen (Einzelkraft und Translation) eine Assoziation gebildet, die sich in unseren Überlegangen unwillkürlich immer wieder geltend macht, wenn man sie nicht durch eine immer wiederholte Erklärung und eine möglichst unzweideutige Sprechweise ausdrücklich abschneidet.

* Vergl. den schon genannten Artikel von Abraham in Bd. 4 der math. Encyklopädie, Art. 14 (Nr. 11 daselbst). 
selben Art, die sich ans den (20), bez. (21) durch „Polarisieren“ ergeben. Ich will hier nur die Polaren der Ausdrücke (21) betrachten:

$$
\left\{\begin{array}{l}
p u^{\prime}+q v^{\prime}+r w^{\prime}+p^{\prime} u+q^{\prime} v+r^{\prime} w, \\
X L^{\prime}+Y M^{\prime}+Z N^{\prime}+X^{\prime} L+Y^{\prime} M+Z^{\prime} N .
\end{array}\right.
$$

Indem dieselben auch ihrerseits Skalare zweiter Art sind, folgt:

Satz I. Die

$\sin d z u$ den

$$
p, q, r, u, v, w
$$

$$
u, v, w, p, q, r
$$

und ebenso natïrlich die

$z u$ den

$$
X, \quad Y, \quad Z, L, \quad M, \quad N
$$

$$
L, \quad M, \quad N, \quad X, Y, Z
$$

bei Bewegungen direkt kontragredient, bei Umlegungen kontragredient mit Zeichenwechsel.

Dem entgegen betrachte man nun den Ausdruck, der sich nach Analogie von (22) bilinear aus den Koordinaten zweier SehraubengröBen verschiedener Art zusammensetzt:

$$
X u+Y v+Z w+L p+M q+N r .
$$

Es folgt sofort, daB derselbe nicht nur bei Bewegungen, sondern (wegen seines Verhaltens bei Inversion) auch bei Umlegungen durchaus ungeändert bleibt; er ist ein Skalar erster Art. Daher kommt:

Satz II. Die

sind $\approx u$ den

$$
X, \quad Y, \quad Z, \quad L, \quad M, \quad N
$$

$$
u, v, w, p, q, r
$$

sowohl bei Bewegungen wie bei Umlegungen schlechtweg kontragredient.

Durch diesen Satz dürfte die Zusammengehörigkeit der beiden Arten von Schraubengrößen in einfachster Weise bezeichnet sein. Verbinden wir ihn mit Satz I, so fallen wir auf die Analogie der zweierlei SchraubengröBen zurück, die der Gegenstand des vorigen Paragraphen war. Dieselbe mag hier folgendermaßen ausgesprochen werden:

Satz III. Die

sind den

$$
X, \quad Y, \quad Z, \quad L, \quad M, \quad \mathcal{N}
$$

$$
p, q, \quad r, u, v, w
$$

bei Bewegungen direkt kogredient, bei Umlegungen kogredient mit Zeichenwechsel.

Die in Rede stehende Analogie folgt hier also aus dem Umstande, dab vermöge des besonderen, durch Satz I festgelegten Verhaltens der Schraubenkoordinaten $p, q, r, u, v, w$ die zu ihnen kontragredienten GröBen 
$X, Y, Z, L, M, N$ zugleich in dem durch Satz III festgelegten Sinne kogredient sind. Hiermit dürfte der algebraische Grundgedanke dieser Beziehnng so klar herausgearbeitet sein, als überhaupt möglich ist. Wir können diesen Gedanken an die Spitze der Schraubentheorie rücken, wenn wir uns das invariante Verhalten des Ausdrucks (23), bez. der Ausdrücke (22), direkt aus ihrer geometrisch-mechanischen Bedeutung klar machen. Dies ist, was ich in meiner wiederholt genannten Notiz in Bd. 4 der Math. Annalen im Auge hatte. Im gegenwärtigen Zusammenhange läßt sich die Sache folgendermaBen präzis darstellen:

1. Man interpretiere die $X, Y, Z, \cdots$ als die Koordinaten eines Systems kontinuierlich wirkender Kräfte. Dann bedeutet der Ausdruck (23) multipliziert mit $d t$, also das Produkt:

$$
(X u+Y v+Z w+L p+M q+N r) d t
$$

die Arbeit, welche das Kräftesystem bei Eintritt der unendlich kleinen Bewegung $u d t, v d t, w d t, \cdots$ leistet, und ist eben darum ein Shalar erster Art.

2. Dagegen haben die Ausdrücke (22) vermöge ihrer geometrischen Bedeutung von rorneherein den Charakter ron Skalaren zweiter Art. Es genügt, dies hier an dem Beispiele zweier Kräftesysteme nachzuweisen, die sich auf Einzelloäfte reduzieren lassen. Wir setzen dementsprechend

und analog

$$
X_{1}=\frac{P_{1}}{l_{1}}\left(x_{1}-x_{1}\right), \quad Y_{1}=\frac{P_{1}}{l_{1}}\left(y_{1}-y_{1}\right), \cdots
$$

$$
X_{2}=\frac{P_{2}}{l_{9}}\left(x_{2}-x_{2}{ }^{\prime}\right), \quad Y_{2}=\frac{P_{2}}{l_{2}}\left(y_{2}-y_{2}{ }^{\prime}\right), \cdots
$$

Hierdurch verwandelt sich $X_{1} L_{2}+Y_{1} M_{2}+Z_{1} N_{2}+X_{3} L_{1}+Y_{2} M_{1}+Z_{2} N_{1}$ in das Produkt von $\frac{P_{1} P_{2}}{l_{9} l_{1}}$ in die Determinante:

$$
\left|\begin{array}{llll}
x_{1} & y_{1} & z_{1} & 1 \\
x_{1}^{\prime} & y_{1}^{\prime} & z_{1}^{\prime} & 1 \\
x_{2} & y_{2} & z_{2} & 1 \\
x^{\prime} & y_{2}^{\prime} & z_{2}^{\prime} & 1
\end{array}\right|,
$$

die einen sechsfachen Tetraederinhalt vorstellt und gewiB ein Skalar zweiter Art ist.

3. Aus der Nebeneinanderstellung von 1. and 2. ergibt sich nun sofort der Satz III, der das zu beweisende Resultat in präziser Form ausspricht. 
$\S 5$.

\section{Gruppentheoretische Charakterisierung der rerschiedenen Arten von Schraubentheorien.}

Bisher haben wir die Substitutionen, welche die Schraubenkoordinaten $p, q, r, u, v, w$ (um nur von diesen zu reden) bei den Bewegungen und Umlegungen erfahren, nur erst durch das Verhalten der $p, q, \cdots$ bei den erzeugenden Operationen (1), (2), (4) definiert. Es ist ron Interesse, den Inbegriff dieser Substitutionen von den Invarianten

$$
p^{2}+q^{2}+r^{2} \text { und } p u+q v+r w
$$

aus zu charakterisieren. In dieser Hinsicht stelle ich folgenden Satz auf:

Die $p, q, r$ erleiden alle ternären linearen Substitutionen von der Determinante +1 , welche $p^{2}+q^{2}+r^{2}$ ungeändert lassen, die $p, q, r, u, v, w$ zusammen aber alle senären linearen Substitutionen von der Determinante \pm 1 , welche $p u+q v+r w$ beaiehungsweise in $\pm(p u+q v+r w)$ überführen.

Der erste Teil dieses Satzes (der sich auf die ternären Substitutionen der $p, q, r$ bezieht) braucht nach den Angaben, die wir über das Verhalten der $p, q, r$ bei den erzeugenden Operationen machten, nicht weiter erläutert zu werden; er bringt nur die bekannte Beziehung der Drehungen um den Koordinatenanfangspunkt $O$ zu den ternären orthogonalen Substitutionen zum Ausdruck. Sei nun irgend eine ternäre orthogonale Substitution der $p, q, r$ von der Determinante +1 als Teil einer senären Substitution der $p, q, r, u, v, w$ von der Determinate \pm 1 vorgelegt, welche $(p u+q v+r w)$ bez. in $\pm(p u+q v+r w)$ verwandelt. Wir kombinieren sie mit einer Drehung um $O$, welche die $p, q, r$ zu ihren Anfangswerten zurückführt (und übrigens für die $u, v, w$ nach den Angaben von $\S \mathbf{2}$ genau dieselbe ternäre Substitution von der Determinante +1 ergibt, wie für die $p, q, r$ selbst, so daB der Wert von $p u+q v+r w$ und der Wert der senären Substitutionsdeterminante dabei ungeändert bleibt). Wir ziehen ferner nötigenfalls eine Inversion heran, um zu erreichen, dab $p u+q v+r w$ seinem ursprünglichen Werte direkt gleich wird; dabei erhält die senäre Substitutionsdeterminante von selbst den Wert +1 . Die so vereinfachte Substitution hat jetzt (weil $p u+q v+r w$ in sich selbst übergehen soll) notwendig die Form

$$
\left\{\begin{array}{l}
p_{1}=p, \quad u_{1}=u-C q+B r, \\
q_{1}=q, \quad v_{1}=v-A r+C p, \\
r_{1}=r, \quad v_{1}=w-B p+A q,
\end{array}\right.
$$

wo einzig die $A, B, C$ noch willkürlich sind. Eine solche Substitution 
stellt aber nach (11), §2, eine Translation dar. Also unsere anfängliche Substitution ergibt eine Translation, wenn wir sie mit einer geeigneten Rotation und eventuell einer Inversion verbinden, - sie stell daher von Hause aus entweder eine Bewegung oder eine Vmlegung dar, was zu beweisen war.

So viel über die Substitutionen der $p, q, r, u, v, w$. Die Substitutionen der $X, Y, Z, L, M, N$ ergeben sich von da aus sofort, wenn wir nar festhalten, dab sie zu den $u, v, w, p, q, r$ kontragredient sind.

Mit dieser Festlegang der beiderlei Substitutionsgruppen ist nach den Grundsätzen meines Erlanger Programms die zugehörige Schraubentheorie vollkommen charakterisiert.

Wir schreiten nach dem oben Gesagten zur Ballschen Schraubentheorie im engern Sinne, indem wir nur die Verhältnisse $p: q: r: u: v: w$ beziehungsweise $X: Y: Z: L: M: N$ in Betracht ziehen (wobei der Unterschied zwischen den Schraubengrößen der beiden Arten wegfällt). Die $p: q: r: u: v: w$ (um nur von diesen zu sprechen) erleiden solche (und alle solchen) linearen Substitutionen, bei denen die Gleichungen

$$
p^{2}+q^{2}+r^{2}=0 \text { und } p u+q v+r w=0
$$

in sich übergehen, der Parameter $\frac{p u+q v+r w}{p^{2}+q^{2}+r^{2}}$ aber entweder überhaupt. ungeändert bleibt oder doch nur sein Zeichen wechselt. Wollen wir neben Bewegungen und Umlegungen auch noch Ähnlichkeitstransformationen in Betracht ziehen, so wird sich $\frac{p u+q v+r w}{p^{2}+q^{2}+r^{2}}$ um einen beliebigen Faktor ändern können; die auf den Parameter bezügliche Einschränkung der Substitution kommt dann in Wegfall.

Die so umgrenzte Ballsche Schraubentheorie ist mit derjenigen Iiniengeometrie, welche das Nullsystem (oder, was dasselbe ist, den linearen Linienkomplex) als Raumelement benutzt, nach dem Klassifikationsprinzip des $\$ 1$ im Wesen identisch. Aber natürlich ist, wenn wir uns so ausdrücken, diejenige Liniengeometrie gemeint, welche die Hauptgruppe räumlicher Änderungen zugrunde legt; ich möchte sie die konkrete Liniengeometrie nennen. Statt dessen ist in meinen eigenen alten Arbeiten (wie auch in der Mehrzahl der seitdem erschienenen deutschen und italienischen Arbeiten) die Liniengeometrie in mehr abstrakter Form behandelt worden, nämlich unter Zugrundelegung der 15 gliedrigen Gruppe, welche einerseits alle projektiven Umformungen unseres Raumes, andererseits aber die dualistischen Umformungen enthält. Für diese abstrakte Liniengeometrie (wie ich sie hier des Gegensatzes halber nennen möchte) gilt dann der Satz, den ich in Bd. 4 der Math. Annalen, pg. 356, aufstellte, daB bei ihr die Gruppe aller derjenigen linearen Substitutionen der $p: q: r: u: v: w$ zugrunde 
liegt, welche die Gleichung $p v+q u+r w=0$ in sich überführen. Die Bezugnahme auf die quadratische Form $p^{2}+q^{2}+r^{2}$ ist einfach weggefallen.

Mit der so gegebenen Entgegenstellung der zugehörigen Gruppe dürfte die Beziehung meiner eigenen alten Arbeiten und beispielsweise des Werkes von Sturm über Liniengeometrie*) zu denjenigen von Ball mit aller Schärfe gegeben sein. Auf Einzelheiten einzugehen ist hier nicht der Ort.

\section{$\S 6$.}

\section{Lineare Schraubensysteme.}

Nachdem solcherweise die Grundlagen der Schraubentheorie festgelegt sind, mögen wir mit Ball dazu übergehen, die linearen Systeme von Schrauben zu studieren, d. h. die Mannigfaltigkeiten solcher Schrauben, deren Koordinaten sich aus den Koordinaten von 2, 3, 4, 5 Schrauben mit Hilfe einer entsprechenden Zahl veränderlicher Parameter homogen linear zusammensetzen lassen. Bei der bezüglichen Diskussion beschränkt sich Ball im wesentlichen auf die Besprechung der allgemeinen Fälle oder zieht doch nur Beispiele ron Spezialfällen heran. Es scheint aber erwünscht, die Diskussion systematisch durchzufïhren.*)

Ich will dies hier für die zweigliedrige Schar skizzieren, beschränke mich aber dabei der Kürze halber darauf, nur die Verhältnisse der sechs Koordinaten in Betracht zu ziehen. Sei dementsprechend:

$$
\varrho p=\lambda_{1} p_{1}+\lambda_{2} p_{2}, \quad \varrho q=\lambda_{1} q_{1}+\lambda_{2} q_{2}, \cdots, \quad \varrho w=\lambda_{1} w_{1}+\lambda_{2} w_{2},
$$

unter o, einen Proportionalitätsfaktor verstanden. Es erleichtert die Ausdrucksweise, wenn wir die so definierten $p: q: \cdots: w$ als homogene Punktkoordinaten in einem Raume von fünf Dimensionen bezeichnen. Die Formeln (25) repräsentieren dann in diesem Raume eine gerade Linie, und es wird sich darum handeln, die sämtlichen Geraden, die es in unserem fünfdimensionalen Raume gibt, nach ihrer Beziehung zu den beiden quadratischen Mannigfaltigkeiten $p^{2}+\dot{q}^{2}+r^{2}=0$ und $p u+q v+r w=0$ zu studieren, resp. zu klassifizieren. Dabei wird sich unsere Aufmerksamkeit in erster Linie auf die Schnittpunkte richten, welche unsere Gerade mit diesen Mannigfaltigkeiten gemein hat. Die Schnittpunkte mit jeder der beiden Mannigfaltigkeiten können getrennt sein, zusammenfallen oder wn-

*) Die Gebilde ersten and $z$ weiten Grades der Liniengeometrie in synthetischer Behandlung, 3 Teile, Leipzig 1892-1896.

*) In ähnlichem Sinne änbert sich Hr. Study auf pg. 226-228 der (bis jetzt allein erschienenen) ersten Lieferung seiner Geometrie der Dynamen (Leipzig, 1901) und stellt für die demnächst erscheinende zweite Lieferung weitergehende Entwicklungen in Aftssicht. 
bestimmt werden. AuBerdem können die Schnittpunkte, welche die gerade Linie mit der einen Mannigfaltigkeit gemein hat, mit denen, die sie mit der anderen Mannigfaltigkeit gemein hat, teilweise oder ganz koinzidieren. Des weiteren möge man Realitätsunterschiede heranziehen. Hiernach ergibt sich eine von vornherein übersehbare Reihe von Fallunterscheidungen, die nicht nur mit leichter Mühe aufgezählt, sondern ebensowohl nach ihrer schraubentheoretischen Bedeutung diskutiert werden können. Jeder Geometer, der mit algebraischen Betrachtungen in mehrdimensionalen Räumen einigermaßen vertraut ist, wird dies ohne weiteres ausführen; es scheint unnötig, hierbei noch länger $\mathrm{zu}$ verweilen.

Immerhin wird es gut sein, einen Unterschied hervorzuheben, den der geschilderte Ansatz den Ballschen Entwicklungen gegenüber zeigt. Ball berücksichtigt prinzipiell nur die reellen Vorkommnisse, hier dagegen wird reell und imaginär zunächst als gleichwertig betrachtet und die Frage nach den Realitätsverhältnissen erst zum Schlusse eingeführt. Um an einem Beispiel den Vorteil zu zeigen, den das letztere Verfahren haben kann, betrachten wir die Regelfläche, welche von den Achsen der Schrauben (25) gebildet wird, das sogenannte Zylindroid. Nach Ball ist dasselbe im allgemeinen von der dritten Ordnung; wenn aber die komponierenden Schrauben $p_{1}, q_{1}, r_{1}, \cdots$ und $p_{2}, q_{2}, r_{2}, \cdots$ sich auf zwei Rotationen reduzieren, deren Achsen sich schneiden, so artet es in dasjenige ebene Strahlbüschel aus, dem die Achsen angehören. Statt der Fläche von der dritten Ordnung haben wir dann also eine von der ersten. Wie kommt diese Ausartung zustande? Wenn wir das Imaginäre mitnehmen, finden wir zunächst, daB es Rotationen mit unbestimmter Achse gibt (es sind diejenigen Schraubenbewegungen, bei denen der durch Formel (19') gegebene Parameter den Wert $\frac{0}{0}$ erhält). Dieselben lassen nämlich alle Minimallinien fest, welche durch einen festen Punkt des Kugelkreises in einer festen Tangentenebene desselben verlauten, also ihrerseits ein Strahlbüschel bilden. Solcher Rotationen treten nun im vorliegenden Spezialfalle unter der Schar (25) zwei auf, entsprechend den beiden Minimallinien, die unter den Strahlen des Ballschen Strahlbüschels enthalten sind. Die Folge ist, daß sich von dem Zylindroid zwei imaginäre Ebenen abtrennen, nämlich die beiden Ebenen, welche sich durch die Normale zum Ballschen Strahlbüschel und die beiden Minimallinien desselben legen lassen. Der Rest, eben das Ballsche Strahlbüschel, ist dann natürlich von der ersten Ordnung. Der Leser muß entscheiden, ob der Gewinn an Einsicht, der hier and in ähnlichen Fällen resultiert, ein Äquivalent für die weitläufigere Vorbereitung ist, die erforderlich scheint, wenn man in der Geometrie mit imaginären Elementen bequem und sicher opezieren will. 
Übrigens möchte ich nicht minder eine Ausgestaltung der Theorie der linearen Schraubensysteme nach der eigentlich mechanischen Seite hin in Anregung bringen. Die Diskussion der linearen Schraubensysteme, von der ich gerade sprach, versieht uns mit einer endlichen Zahl unterschiedener Fälle der Beweglichkeit eines starren Körpers im Unendlich-Kleinen; es kann sich dabei der Reihe nach um 2, 3, 4, 5 Grade der Freiheit handeln. Nun findet man in der Natural Philosophy ron Thomson and Tait (2. ed., vol. I, p. 155 (Nr. 201)) einen einfachen Mechanismus beschrieben, vermöge dessen man einem starren Körper fünf Grade der Beweglichkeit im Unendlich-Kleinen in allgemeinster Weise erteilen kann: der Körper ist um eine Schraubenspindel drehbar, die mit Hilfe zweier aneinander geketteter Hookescher Schlüssel an ein Postament befestigt ist. Ich stelle die Aufgabe, die sämtlichen gemäß unserer Diskussion zu unterscheidenden reellen Fälle infinitesimaler Beweglichkeit eines starren Körpers durch möglichst einfache Mechanismen zu realisieren.

Eine letzte Bemerkung zur Theorie der linearen Schraubensysteme möge wieder nach Seite der Gruppentheorie liegen. Camille Jordan hat bekanntlich zuerst alle kontinuierlichen und diskontinuierlichen Gruppen aufgestellt, die sich aus den reellen Bewegungen des Raumes bilden lassen.*) Unter diesen interessieren uns hier nur die kontinuierlichen Gruppen. Man findet dieselben bei Study im 39. Bande der Math. Annalen, p. 486-487, übersichtlich zusammengestellt und geometrisch charakterisiert; eine Tabelle der zugehörigen unendlich kleinen Bewegungen gibt Lie in Bd. III seiner Theorie der Transformationsgruppen (Leipzig, 1893), p. 385. .Ich nenne hier von diesen Groppen nur die einfachsten, nämlich: a) die Gesamtheit aller $\infty^{3}$ Translationen,

b) die Gesamtheit aller $\infty^{4}$ Bewegungen, die einen unendlich fernen Punkt (oder, was auf dasselbe hinauskommt, eine unendlich ferne Gerade) festlassen,

c) die Gesamtheit aller $\infty^{3}$ Bewegungen, welche einen im Endlichen gelegenen Punkt festlassen,

d) die Gesamtheit aller $\infty^{3}$ Bewegungen, welche eine im Endlichen gelegene Ëbene festlassen.

Offenbar empfiehlt es sich, die Mechănik solcher starrer Körper, welche die Beweglichkeit einer dieser Untergruppen haben, gesondert $z \mathbf{u}$ bearbeiten (wie dies für den Körper mit im Endlichen gelegenem festem Punkt von jeher geschehen ist). Die unendlich kleinen Bewegangen jeder solchen Untergruppe bilden aber ein lineares Schraubensystem, und die so entstehenden linearen Schraubensysteme heben sich also vor anderen 
durch ihre Wichtigkeit für die Mechanik hervor; ich werde sie lineare Schraubensysteme von selbständiger gruppentheoretischer Bedeutung nennen. Indem ich das Koordinatensystem in geeigneter Weise wähle, bekomme ich in den Fällen a) bis d) für die Koordinaten

$$
p, q, r, u, v, w
$$

der betreffenden Schrauben folgende Werte:
a) $0,0,0, \lambda_{1}, \lambda_{2}, \lambda_{3}$
b) $0,0, \lambda_{1}, \lambda_{2}, \lambda_{3}, \lambda_{4}$
c) $\lambda_{1}, \lambda_{2}, \lambda_{3}, 0,0,0$
d) $0,0, \lambda_{1}, \lambda_{2}, \lambda_{3}, 0$.

Hier sind die $\lambda_{1}, \lambda_{2}, \cdots$, wie in (25), beliebig veränderliche Parameter. Man sollte jedes einzelne der so gewonnenen linearen Schraubensysteme genau so für die Mechanik der ihm zugehörigen endlichen Bewegungen benutzen, wie dies sofort mit dem System c) für die Drehung eines Körpers um einen festen Punkt und hernach mit der Gesamtheit aller Schrauben für den in allgemeinster Weise beweglichen starren Körper geschehen wird.

$$
\S 7 .
$$

Übergang zur Kinetik. Unterscheidung holonomer und nicht holonomer Differentialausdrücke bez. Differentialbedingungen.

DaB für $n \geqq 2$ nicht jeder Differentialausdruck

$$
\Sigma \varphi_{i}\left(x_{1}, \cdots, x_{n}\right) d x_{i}
$$

ein exaktes Differential $d F$ einer Funktion von $x_{1}, \cdots, x_{n}$ ist, und daB für $n \geqq 3$ nicht jede Differentialbedingung

$$
\Sigma \varphi_{i} d x_{i}=0
$$

mit einer Gleichung $d F=0$ gleichbedeutend ist, ist bekannt genug; die Klassifikation der verschiedenen in dieser Hinsicht vorliegenden Möglichkeiten wird in der Theorie des "Pfaffschen Problems“ entwickelt. Wir sprechen nach der Ausdrucksweise von Hertz in allen den Fällen, wo der Differentialausdruck oder die Differentialbedingung nicht durch ein einfaches $d F$ ersetzt werden kann, von einem nicht holonomen Differentialausdruck, bez. einer nicht 7oolonomen Differentialbedingung.

In der Mechanik liegt die Sache, allgemein zu reden, nun merkwürdigerweise so, daB man zwar von je AnlaB hatte, nicht holonome Differentialausdrücke und -bedingungen in Betracht zu ziehen, dab man 
aber erst in den letzten Jahren angefangen hat, diesem Umstande besondere Aufmerksamkeit zuzuwenden.*)

Was zunächst nicht holonome Differentialausdrücke angeht, so treten dieselben in unsere jetzige Betrachtung dadurch ein, dab bereits die Koordinaten $p d t$, qdt, rdt einer unendlich kleinen Drehung um 0 , and umsomehr die Schraubenkoordinaten $p d t, q d t, \cdots, w d t$ einer beliebigen unendlich kleinen Verrückung eines starren Körpers nicht holonome Verbindungen der Differentiale der 3 oder 6 endlichen Parameter sind, durch welche man die Lage des Körpers in den beiden Fällen festlegen mag; wir werden hierfür sogleich noch explizite Formeln geben.

Was aber nicht holonome Bedingungsgleichungen betrifft, so bilden dieselben nicht etwa einen Ausnahmefall, sondern treten bei den mechanischen Vorgängen, die wir täglich beobachten, auBerordentlich häufig auf. So macht Hertz in seinem Werke äber die Prinzipien der Mechanik**) darauf aufmerksam, daß eine Kugel, die auf einer Fbene rollt, das Beispiel eines mechanischen Systems von 5 Freiheitsgraden abgibt, das an eine nicht holonome Bedingungsgleichung gebunden ist. Noch einfacher ist vielleicht das Beispiel eines auf horizontaler Ebene beweglichen Wagens oder Schlittens, der (wegen der Reibung an der Unterlage) immer nur in Richtung seiner Achse fortschreiten kann; wir haben hier die nicht holonome Bedingungsgleichung $d y-\operatorname{tang} \vartheta \cdot d x=0$, unter $\vartheta$ das Azimut der Achse verstanden. Wir schlieBen, daß die Betrachtung nicht holonomer Bedingungsgleichungen in der Mechanit nichts Künstliches ist, sondern von vorneherein mit in Betracht gezogen werden muß, wenn anders wir die Bewegungsvorgänge der uns umgebenden Wirklichkeit verstehen wollen.

Wir werden daher die nicht holonomen Bedingungsgleichungen im folgenden immer mit erwähnen. Bei Ball geschieht dies nicht und braucht nicht zu geschehen, da Ball seine Betrachtungen von vorneherein in der Weise auf unendlich kleine Ortsänderungen einschränkt, daB er nur die ersten Potenzen der Differentiale beibehält. Infolgedessen kann Ball auch den starren Körper, der irgend $k$ Differentialbeziehungen vom Typus (26) unterworfen ist, kurzweg als ein mechanisches System von $(6-k)$ Freiheitsgraden bezeichnen. Dies würde im Falle endlicher Bewegungen nicht richtig sein: die rollende Kugel vermag trotz der nicht holonomen Bedingung, der ihre infinitesimalen Bewegungen unterworfen sind, $\infty^{5}$ Lagen anzunehmen, ebenso der auf der $(x, y)$-Ebene bewegliche Wagen sämtliche $\infty^{3}$ Lagen $(x, y, \vartheta)$.

*) Vergl. verschiedene Stellen in VoB, Die Prinzipien der rationellen Mechanik (Encyklopädie der Math. Wiss. IV, 1), insbesondere Nr. 38 daselbst.

*) Einleitung, p. 23. 
$\S 8$.

\section{Über die Verwendnng der Geschwindigkeitskoordinaten $p, q, r$ in der Kinetik des starren Körpers mit festem Punkt.}

Ehe wir zur Verwendung der Schraubenkoordinaten $p, q, r, u, v, w$ in der Kinetik beliebiger starrer Körper schreiten, mögen wir die Verwendung der $p, q, r$ in der Kinetik des starren Körpers mit festem Punkt betrachten. Es handelt sich dabei 2war im Prinzip um lauter bekannte Dinge, aber man findet dieselben nicht überall in der einfachen und präzisen Form beisammen, die wir ihnen hier geben wollen, und die sich hernach unmittelbar auf die Schraubenkoordinaten $p, q, r, u, v, w$ überträgt. Den einzelnen Angaben Beweise hinzuzufügen, wird kaum nötig sein; ich verweise wegen der etwaigen Ableitung der Resultate, sofern deutsche Literatur in Betracht gezogen werden soll, am liebsten auf die von Sommerfeld und mir herausgegebenen Vorlesungen über die Theorie des Kreisels (Teil I, Leipzig 1897); insbesondere geschieht dort (pag. 138ff.) die Herleitung der Eulerschen Bewegungsgleichungen (im AnschluB an die ursprüngliche Entwicklung von Hayward) genau so, wie es im folgenden skizziert wird.

1. Zusammenhang der $p, q, r$ mit den Geschwindigkeitskoordinaten $\varphi^{\prime}, \psi^{\prime}, \vartheta^{\prime}$.

Wir nehmen ein im Körper festes Koordinatensystem $X Y Z$ und ein im Raume festes $x y z$ (mit gemeinsamem Anfangspunkt), deren gegenseitige Beziehung wir durch irgend drei Parameter, für welche wir hier wegen ihres elementaren Charakters die Eulerschen Winkel $\varphi, \psi$, $\vartheta$ nehmen wollen, festlegen (Kreisel, pg. 19). Der Übergang von der Lage $\varphi, \psi, \vartheta$ zur Lage $\varphi+\varphi^{\prime} d t, \psi+\psi^{\prime} d t, \vartheta+\vartheta^{\prime} d t$ sei äquivalent mit einer Drehung durch $p d t, q d t, r d t$ um die Achsen des $X Y Z$-Systems in seiner den Parameterwerten $\varphi, \psi, \vartheta$ entsprechenden Lage. Die Nebeneinanderstellung der bezüglichen Formeln ergibt dann folgenden Zusammenhang zwischen $\operatorname{den} p, q, r$ und den $\varphi, \psi, \vartheta$, bez. $\varphi^{\prime}, \psi^{\prime}, \vartheta^{\prime}$ (Kreisel, pg. 45):

$$
\left\{\begin{array}{l}
p=\vartheta^{\prime} \cos \varphi+\psi^{\prime} \sin \vartheta \sin \varphi, \\
q=-\vartheta^{\prime} \sin \varphi+\psi^{\prime} \sin \vartheta \cos \varphi, \\
r=\varphi^{\prime}+\psi^{\prime} \cos \vartheta .
\end{array}\right.
$$

Man erkennt, dab die $p, q, r$ nicht-holonome Verbindungen der $\varphi^{\prime}, \psi^{\prime}, \vartheta^{\prime}$ sind. Die Folge ist, $\mathrm{daB}$ ich in den Bewegungsgleichungen des starren Körpers zwar die $\varphi^{\prime}, \psi^{\prime}, \vartheta^{\prime}$ gern durch die $p, q, r$ ersetzen kann, daß ich aber daneben zur Lagenbestimmung des Körpers die $\varphi, \psi, \vartheta$ festhalten 
muB, die dann mit den $p, q, r$ durch die Gleichangen (27), welche ich die kinematischen Gleichungen nenne, verbunden sind.

\section{Kraftkoordinaten.}

Hat man bei irgend einem mechanischen System bestimmte Geschwindigkeitskoordinaten (hier also die $p, q, r$ ) ausgewählt, so hat man als Koordinaten der kontinuierlich wirkenden Kräfte allgemein diejenigen GröBen zu nehmen, mit denen multipliziert die Koordinaten der unendlich kleinen Bewegung in den Ausdruck für die Arbeit eingehen. Im vorliegenden Falle haben wir für die Arbeit nach (24) oben (indem die $u, v, w$ verschwinden):

$$
d A=(L p+M q+N r) d t
$$

wir werden also das Kräftesystem, das am starren Körper angreift, durch seine Drehmomente $L, M, N$ um die Achsen des im Körper festen Koordinatensystems festzulegen haben. Genau so werden wir als Koordinaten einer Stoßkraft ihre bezüglichen Drehmomente wählen, wie wir nieht weiter ansführen.

3. Aufstellung der kinetischen Gleichungen für die $p, q, r$.

Die Aufstellung der eigentlichen Bewegungsgleichungen für die $p, q, r$ (der Eulerschen Bewegungsgleichungen) erfolgt nun am kürzesten folgendermaBen:

a) Man drücke die lebendige Kraft des rotierenden Körpers durch die $p, q, r$ aus. Als Einheit der Masse ist dabei natürlich, auf Grond unserer früheren Verabredungen, diejenige zu wählen, die bei Einwirkung einer kontinuierlichen Kraft von der GröBe 1 in der Zeiteinheit die Geschwindigkeit 1 erhält. $\mathrm{Da}$ sich die $p, q, r$ auf ein im Körper festes Koordinatensystem beziehen, erhält man eine quadratische Form derselben mit konstanten Koeffizienten

$$
T=\frac{1}{2}\left(A p^{2}+B q^{2}+C r^{2}+2 D q r+2 E r p+2 F p q\right)
$$

b) Hierauf bilde man die Koordinaten $L, M, N$ des sogenannten "Impulses", d. h. desjenigen Systems von Stobkräften, welches imstande wäre, den in seiner augenblicklichen Lage ruhend gedachten Körper instantan in den Geschwindigkeitszustand $p, q, r$ zu versetzen. Nach den Grundgesetzen der Kinetik, die in der sogenannten ,ersten Zeile der Lagrangeschen Gleichungen" ihren Ausdruck finden, erhält man dieselben aus $T$ durch Differentiation nach den entsprechenden Geschwindigkeitskoordinaten. Die Formeln sind:

$$
L=\frac{\partial T}{\partial p}, \quad M=\frac{\partial T}{\partial q}, \quad N=\frac{\partial T}{\partial r} .
$$


c) Von hier aus erhält man nun die gesuchten kinetischen Gleichungen, indem man überlegt, dab sich die Koordinaten $L, M, N$ des Impulses während des Zeitelementes $d t$ ans zwei Gründen um unendlich kleine Beträge abändern.

Erstlich dadurch, daß an unserem Körper von außen gegebenenfalls ein System kontinuierlich wirkender Kräfte angreift. Wir nennen die Koordinaten dieses Systems (d. h. seine Drehmomente um die $X$-, $Y$-, $Z$-Achse) $\Lambda, M, N$. Die von hier aus resultierenden Änderungen der $L, M, N$ sind:

$$
d^{\prime} L=\Lambda d t, \quad d^{\prime} M=\mathrm{M} d t, \quad d^{\prime} N=\mathrm{N} d t
$$

Zweitens aber ändern sich die $L, M, N$ dadurch, dab sich das Koordinatensystem $X Y Z$, auf welches sie bezogen sind, während des Zeitelementes $d t$ gegen seine ursprüngliche Lage um $p d t, q d t, r d t$ gedreht hat. Wir können ebensowohl sagen, daß wir den Raum (und also den im Raume feststehenden Impulsvektor) gegen das Koordinatensystem der $X, Y, Z$ um - pdt, - qdt, - rdt gedreht haben. Dies gibt als Änderungen der $L, M, N$ : (31) $d^{\prime \prime} L=(r M-q N) d t, \quad d^{\prime \prime} M=(p N-r L) d t, \quad d^{\prime \prime} N=(q L-p M) d t$.

Die Gesamtänderung $\operatorname{der} L, M, N$ ist die Summe der Änderungen (30), (31); daher kommt, wenn wir noch durch $d t$ dividieren:

$$
\left\{\begin{array}{l}
\frac{d L}{d t}=(r M-q N)+\Lambda \\
\frac{d M}{d t}=(p N-r L)+M \\
\frac{d N}{d t}=(q L-p M)+\mathrm{N}
\end{array}\right.
$$

und dieses sind die gesuchten kinetischen Gleichungen. Die $\Lambda, M, N$ werden dabei zunächst als Funktionen der $\varphi, \psi, \vartheta$ anzusetzen sein.

\section{Bemerkungen zu den gewonnenen Gleichungen.}

Schlieblich haben wir zur Darstellung der Bewegung die Gleichungen (27), (28), (29), (32), wo wir noch die aus (29) folgenden Werte der $L, M, N$ in die (32) eintragen können. Wir haben dann 6 Differentialgleichungen erster Ordnung für die $\varphi, \psi, \vartheta, p, q, r$. Ist insbesondere irgend eine (holonome oder nicht holonome) Bedingungsgleichung für die $\varphi^{\prime}, \psi^{\prime}, \vartheta^{\prime}$ gegeben, so wird sich diese in eine lineare Gleichung für die $p, q, r$ umsetzen lassen (deren Koeffizienten, allgemein zu reden, Funktionen $\operatorname{der} \varphi, \psi, \vartheta$ sind):

$$
P p+Q q+R r=0 \text {. }
$$

Es werden dann in den $\Lambda, M, N$ neben Gliedern, welche sich auf die anderweitigen äuBeren Kräfte beziehen, Terme folgender Form auftreten: 


$$
-\lambda P,-\lambda Q,-\lambda R,
$$

unter $\lambda$ einen Lagrangeschen Multiplikator verstanden, der so zu bestimmen ist, daB die Gleichung (33) fortgesetzt erfüllt ist.

\section{$\S 9$.}

\section{Fortsetzung. Fälle, wo die $p, q, r$ wie Lagrangesche Geschwindigkeitskoordinaten gebrancht werden können.}

Die Betrachtungen, welche wir im vorigen Paragraphen unter 3. gaben, sind wesentlich durch den Umstand veranlabt, daß die $p, q, r$ keine Lagrangeschen Geschwindigkeitskoordinaten, d. h. keine holonomen Verbindungen der $\varphi^{\prime}, w^{\prime}, \vartheta^{\prime}$ sind; wir hätten andernfalls nur die „zweite Zeile" der allgemeinen Lagrangeschen Bewegungsgleichungen heranzuziehen brauchen. Es hat daher Interesse, zuzusehen, bei welchen Ansätzen und Problemen der Unterschied der $p, q, r$ und der Lagrangeschen Geschwindigkeitskoordinaten noch nicht hervortritt; wir lösen dadurch aus der allgemeinen Theorie der Rotation eines starren Körpers einen relativ elementaren Teil heraus. In dieser Hinsicht ergibt sich zunächst folgende Zusammenstellung:

1. Die Bedingungsgleichungen, welche gegebenenfalls die Beweglichkeit des Körpers im Enendlich-Kleinen einschränken, sind in den $p, q, r$ ebenso linear, wie in den $\varphi^{\prime}, \psi^{\prime}, \vartheta^{\prime}$ (vergl. Gl. (33)).

2. Der Unterschied verschwindet fermer bei den Fragen der Statik, insofern bei ihnen die $p, q, r$ (und also auch die $L, M, N$ ) darchweg gleich Null zu setzen sind.

3. Er verschwindet endlich in der Stoßtheorie; in der Tat sind die Gleichungen (29), die den Zusammenhang des Impulses mit den erzeugten Geschwindigkeitskoordinaten $p, q, r$ ergeben, ihrer Form nach von dem Umstande, dab die $p, q, r$ nicht bolonome Geschwindigkeitskoordinaten sind, durchaus unabhängig.

Es sind dies einfach diejenigen Teile der Mechanik, welche der Aufstellung der auf kontinuierliche Kräfte bezüglichen Bewegungsgleichungen vorangehen. Hierzu tritt aber, wenn man approximative Rechnung zulassen will, noch ein vierter Punkt. Derselbe liegt vor, wenn man die Theorie der kleinen Schwingungen unseres starren Körpers um eine Gleichgewichtslage behandelt, und dabei die üblichen Vernachlässigungen eintreten läßt. Man nimmt dann nämlich an, daB man die in (32) rechter Hand anftretenden "Glieder zweiter Ordnung", also die $(r M-q N)$ etc., gegen die übrigen Glieder, also die $\frac{d L}{d t}$ und $\Lambda$, etc., vernachlässigen kann. Man erhält solcherweise die vereinfachten Formeln: 


$$
\left\{\begin{array}{l}
\frac{d L}{d t}=\Lambda \\
\frac{d M}{d t}=\mathrm{M} \\
\frac{d N}{d t}=\mathrm{N}
\end{array}\right.
$$

und diese hängen mit dem Ausdruck (28) der lebendigen Kraft in der Tat so zusammen, als wenn die $p, q, r$ Lagrangesche Geschwindigkeitskoordinaten wären.

Es steht überhaupt nichts im Wëge, sofern man Glieder höherer Ordnung vernachlässigen will, die $p, q, r$ nach der Zeit genommenen exakten Differentialquotienten von Funktionen der $\varphi, \psi, \vartheta$ gleichzusetzen. Wir werden eine unendlich kleine Drehung vor uns haben, wenn wir $\vartheta$ und $\varphi+\psi=\chi$ unendlich klein nehmen. Ersetzen wir dementsprechend in (27) $\sin \vartheta$ durch $\vartheta, \cos \vartheta$ durch $1, \psi^{\prime} \cdot \vartheta \operatorname{durch}-\varphi^{\prime} \cdot \vartheta$ und $\varphi^{\prime}+\psi$ durch $\chi^{\prime}$, so kommt:

$$
\left\{\begin{array}{l}
p=\vartheta^{\prime} \cos \varphi-\varphi^{\prime} \cdot \vartheta \sin \varphi=\frac{d(\vartheta \cos \varphi)}{d t} \\
q=-\vartheta^{\prime} \sin \varphi-\varphi^{\prime} \cdot \vartheta \cos \varphi=\frac{d(-\vartheta \sin \varphi)}{d t} \\
r=\chi^{\prime}
\end{array}\right.
$$

Hier sind $\vartheta \cos \varphi,-\vartheta \sin \varphi, \chi$ die unendlich kleinen Winkel, durch welche der Körper von seiner Anfangslage aus um die Achsen $O X, O Y$, $O Z$ gedreht ist.

Die Aufzählung der vorgenannten vier Punkte ist für das Verständnis der Ballschen Schraubenuntersuchungen von unmittelbarster Wichtigkeit. Wir dürfen vorgreifend erwähnen, daß die Schraubenkoordinaten $p, q, r$, $u, v, w$ (wie überhaupt irgend welche nicht-holonome Geschwindigkeitskoordinaten) genau in den entsprechenden vier Fällen ebenfalls wie Lagrangesche Geschwindigkeitskoordinaten behandelt werden können. Und nun trifft es sich so, $\mathrm{daB}$ Ball in seinen ursprünglichen Untersuchungen über die Anwendung der Schraubentheorie auf die Mechanik der starren Körper gerade die vier hiermit bezeichneten Kapitel herausgegriffen hat. Und auch die weitere Frage, die er später in Angriff nabm und von der noch genauer weiter unten die Rede sein soll, die Frage nach den jeweils vorhandenen permanenten Schrauben, läßt sich unter denselben Gesichtspunkt bringen. Dies ist gewiß nicht,zufällig, sondern wohlbedacht, entsprechend der Auffassung, daB es in der Mechanik vor allen Dingen daranf ankommt, sich die jeweils einfachsten Beziehungen und Vorgänge klar zu machen. 


\section{$\S 10$.}

\section{Verwendung der Sehraubenkoordinaten für allgemeine Kinetik der starren Körper.}

Das in $\$ 7$ Entwickelte läßt sich nun Schritt für Schritt auf die Frage nach der Verwendung der Schraubenkoordinaten für die allgemeine Kinetik der starren Körper übertragen.

1) Wir fixieren die jeweilige Ortsänderung des starren Körpers durch irgend 6 Parameter, etwa so, daß wir wieder ein im Körper festes Koordinatensystem $X Y Z$ einführen und dessen Lage gegen ein im Raume festes System $x y z$ durch die Verschiebungskomponenten $\xi, \eta, \xi$ des Anfangspunktes und die drei Eulerschen Winkel $\varphi, \psi, v$ festlegen (was freilich sehr unsymmetrische Formeln ergibt). Die auf das Koordinatensystem XYZ bezüglichen Schraubenkoordinaten $p, q, r, u, v, w$ der instantanen Geschwindigkeit werden sich dann in folgender Weise als lineare, nicht holonome Verbindungen der $\xi^{\prime}, \eta^{\prime}, \zeta^{\prime}, \varphi^{\prime}, \psi^{\prime}, \vartheta^{\prime}$ darstellen:

$$
\left\{\begin{array}{c}
p=\vartheta^{\prime} \cos \varphi+\psi^{\prime} \sin \vartheta \sin \varphi, \quad q=-\vartheta^{\prime} \sin \varphi+\psi^{\prime} \sin \vartheta \cos \varphi \\
r=\varphi^{\prime}+\psi^{\prime} \cos \vartheta \\
u=\xi^{\prime}(\cos \varphi \cos \psi-\cos \vartheta \sin \varphi \sin \psi) \\
\quad+\eta^{\prime}(\cos \varphi \sin \psi+\cos \vartheta \sin \varphi \cos \psi)+\xi \sin \vartheta \sin \varphi \\
v=\xi^{\prime}(-\sin \varphi \cos \psi-\cos \vartheta \cos \varphi \sin \psi) \\
+\eta^{\prime}(-\sin \varphi \sin \psi+\cos \vartheta \cos \varphi \cos \psi)+\xi^{\prime} \sin \vartheta \cos \varphi \\
w=\xi^{\prime} \sin \vartheta \sin \psi-\eta^{\prime} \sin \vartheta \cos \psi+\xi^{\prime} \cos \vartheta
\end{array}\right.
$$

Wir bezeichnen diese Gleichungen wieder als die kinematischen Gleichangen.

2) Um nunmehr zu den kinetischen Gleichungen zu kommen, drücken wir erstlich die lebendige Kraft des Körpers durch die $p, q, r, u, v, w$ aus; wir erhalten eine quadratische Form mit konstanten Koeffizienten:

$$
T=F(p, q, r, u, v, w) \text {. }
$$

Wir berechnen ferner, gemäB der ersten Zeile der Lagrangeschen Gleichungen und dem Ausdruck (24) für die virtuelle Arbeit eines Kräftesystems, die Sehraubenkoordinaten $X, Y, Z, L, M, N$ des zum Geschwindigkeitszustande $p, q, r, u, v, w$ gehörigen Impulses durch die Formeln:

(39) $\quad X=\frac{\partial T}{\partial u}, \quad Y=\frac{\partial T}{\partial v}, \quad Z=\frac{\partial T}{\partial w}, \quad L=\frac{\partial T}{\partial p}, \quad M=\frac{\partial T}{\partial q}, \quad N=\frac{\partial T}{\partial r}$.

Wir überlegen endlich, daß diese Impulskoordinaten während des Zeitelementes $d t$ ans zwei Gründen Änderungen erfahren, die sich superponieren, nämlich durch die von außen auf den Körper wirkenden Kräfte, die zusammengenommen die Koordinaten

$$
\equiv, H, Z, \wedge, M, N
$$


ergeben mögen, und durch die Bewegung des im Körper festen Koordinatensystems mit dem Körper. Fon hier aus erhalten wir:

$$
\begin{cases}\frac{d X}{d t}=(r Y-q Z)+\equiv, & \frac{d L}{d t}=(w Y-v Z)+(r M-q N)+\Lambda \\ \frac{d Y}{d t}=(p Z-r X)+H, & \frac{d M}{d t}=(u Z-w X)+(p N-r L)+\mathrm{M} \\ \frac{d Z}{d t}=(q X-p Y)+Z, & \frac{d N}{d t}=(v X-u Y)+(q L-p M)+\mathrm{N}\end{cases}
$$

and dies sind die gesuchten kinetischen Gleichungen.

3) An diese Entwicklung schlieBen sich dann genau dieselben Bemerkungen wie in $\S 7$, insbesondere auch, was die Berücksichtigung irgend welcher Bedingungsgleichungen angeht.

\section{$\S 11$.}

\section{Spezielle Ausfiuhrungen zu den Entwicklungen des vorigen Paragraphen.}

Um die Entwicklungen des* vorigen Paragraphen durch spezielle Ausführungen zu belegen, ziehen wir zuvörderst den Fall eines isolierten, frei beweglichen Körpers heran. Die Sache wird dann eminent einfach, verliert aber zugleich einen guten Teil ihrer spezifischen Bedeutung. Wir legen den Anfangspunkt des Koordinatensystems in den Schwerpunkt des Körpers. Die lebendige Kraft (38) nimmt dann bekanntlich folgende einfache Form an:

$$
T=\frac{m}{2}\left(u^{2}+v^{2}+w^{2}\right)+f(p, q, r),
$$

unter $f$ eine quadratische Form der beigesetzten Argumente mit konstanten Koeffizienten verstanden. Die Impulskoordinaten (39) werden daraufhin (42) $\quad X=m u, \quad Y=m v, \quad Z=m w, \quad L=\frac{\partial f}{\partial p}, \quad M=\frac{\partial f}{\partial q}, \quad N=\frac{\partial f}{\partial r}$.

Es nehmen daher die letzten drei Gleichungen (40) folgende einfache Form an:

$$
\left\{\begin{array}{l}
\frac{d L}{d t}=(r M-q N)+\Lambda \\
\frac{d M}{d t}=(p N-r L)+M \\
\frac{d N}{d t}=(p L-p M)+N
\end{array}\right.
$$

Wollen wir nun noch voraussetzen, $\operatorname{da} \beta$ die $\Lambda, M, N$ nur von $\operatorname{den} \varphi, \psi, \vartheta$ (nicht von den $\xi, \eta, \xi$ ) abhängen, so haben wir ersichtlich zur Bestimmung der $p, q, r$, d. h. der Drehung um den Schwerpunkt, genau denselben Ansatz, den man von jeher benutzt hat. Das Eigenaxtige der Schraubentheorie entschwindet; man wird das Problem am einfachsten so weiter behandeln, daB man nach Bestimmung der Drehung um den Schwerpunkt die fort- 
schreitende Bewegang des letzteren direkt bestimmt, d. h. die gewöhnlichen Bewegungsgleichungen für die $\xi, \eta, \xi$ aufstellt. Die Schraubentheorie erleidet hier also so zu sagen einen MiBerfolg. An diesem Mißerfolg mag es liegen, daB sich die Schraubentheorie die groBe Geltung, welche sie zweifellos für die Mechanik der starren Körper besitzt, immer nur erst partiell hat erringen können. Gäbe es in der Mechanik der starren Körper keine anderen Aufgaben, als die gerade besprochenen, so wäre es ïberflüssig, eine besondere Schraubentheorie zu entwickeln.

Es gibt aber andere Aufgaben die Menge. Ich nenne hier die Bewegung eines starren Körpers in einem widerstehenden Mittel (wo die $\Lambda, M, N$ gewiß nicht von den $\varphi, \psi, \vartheta$ allein abhängen), ferner aber die Bewegung eines starren Körpers, der gezwungen ist, auf anderen starren Körpern zu rollen oder zu gleiten.

Ich möchte hier insbesondere auf dasjenige Problem hinweisen, bei welchem die Schraubentheorie bislang die glänzendste Verwendung gefunden haben dürfte, das Problem von der Bewegung des starren Körpers in einer reibungslosen inkompressiblen Flüssigkeit.*) Die lebendige Kraft des aus Körper und Flüssigkeit gebildeten Systems kann in diesem Falle ohne weiteres in der Form (38) angeschrieben werden, woranf die gesamten Entwicklungen des vorigen Paragraphen Platz greifen. Diese Entwicklungen sind in der Tat nichts anderes als eine Transskription der Ansätze, welche Lord Kelvin und Kirchhoff ursprünglich für den Körper in Flüssigkeit gemacht haben; man vergleiche die Darstellung bei Lamb, Hydrodynamics (Cambridge, 1895; Kap. 6), der sich direkt an die Ausdrucksweise der Schraubentheorie anschliebt, sowie das Referat von Love in IV 15 und IV 16 der mathematischen Encyklopädie. Die verschiedenen Formen, welche die lebendige $\mathrm{Kraft} T$ je nach der Symmetrie des in die Flüssigkeit getauchten Körpers annimmt, der jeweilige Zusammenhang zwischen der instantanen Geschwindigkeitsschraube und der Impulsschraube, endlich die resultierende Bewegung des Körpers selbst sind ebenso viele Gegenstände, welche sich auch für eine anschanlich-geometrische Diskussion im Sinne der Ballschen Schraubentheorie vorzüglich eignen dürften. Es würde dies eine direkte und doch nicht triviale Weiterbildung von Poinsots berühmten Untersuchungen über die Rotation eines starren Körpers um einen festen Punkt sein. Hierzu wolle man insbesondere die Arbeit von Minkowski in den Sitzangsberichten der Berliner Akademie von 1888 vergleichen.

7) Leider ist die mathematische Eleganz dieser Untersuchungen kein MaBstab für ihre physikalische Wichtigkeit; vielmehr ist das praktische Geltungsgebiet derselben wegen der in allen Fällen vorhandenen Flüssigkeitsreibung and der bei gröBeren Geschwindigkeiten anftretenden turbulenten Bewegungen ein sehr geringes. 
$\S 12$.

\section{AbschlieBende Bemerkungen über die mechanischen Kapitel des Ballschen Werkes. - Verallgemeinerungen des in $\$ 7$ und $\$ 9$ gegebenen Ansatzes.}

Es wurde bereits in $\S 8$ hervorgehoben, daB die Untersuchungen über die Mechanik der starren Körper, welche Ball in seinem Werke ausführt*), einen übereinstimmenden Charakter zeigen: es handelt sich bei Ball durchweg um solche Fragen, bei denen die Schraubenkoordinaten $p, q, r$, $u, v, w$ der instantanen Geschwindigkeit wie Lagrangesche Geschwindigkeitskoordinaten benutat werden können. Ich habe dies hier nur noch betreffs der letzten Frage, die in $\$ 8$ genannt wurde, der Frage nach den jeweiligen permanenten Schrauben auszuführen. Dies gelingt in einfachster Weise im AnschluB an die kinetischen Gleichungen (40). Man findet nämlich, daB es sich bei Ball dabei um die Aufsuchung solcher Werte der $p, q, r$, $u, v, w$ bez. $\varphi, \psi, \vartheta, \xi, \eta, \xi$ handelt, für welche die rechten Seiten der. kinetischen Gleichungen (40) verschwinden; es bleiben dann die $X, Y, Z$, $L, M, N$ des Impulses und also auch die $p, q, r, u, v, w$ wenigstens für ein Zeitelement konstant, und eben deshalb spricht Ball in einem solchen Falle von einer permanenten Schraube. Als einfache Beispiele möchte ich anführen Staudes permanente Drehachsen eines um einen Punkt rotierenden schweren Körpers (Journal für Mathematik Bd. 113, 1894), sowie Kirchhoffs Theorem, daB bei jedem Körper in einer reibungslosen, inkompressiblen Flüssigkeit bei Abwesenheit äuBerer Kräfte drei zueinander senkrechte Richtungen gleichförmiger Translation existieren. Die sämtlichen Fälle stationärer Bewegung, welche in dem genannten Falle bei dem Körper in Flüssigkeit auftreten können, diskutiert Minkowski l. c. In diesen Beispielen sind zugleich die $p, q, r, u, v, w$ nicht nur zeitweise, sondern dauernd konstant, so daß man von Permanenz der bez. Schrauben im vollen Sinne des Wortes reden kann.

Letzterer Umstand hängt ersichtlich mit der Tatsache zusammen, daB die Drehungen um einen Punkt, wie andererseits die Bewegungen eines freien Körpers eine Gruppe bilden: gehört eine unendlich kleine Bewegung der Gruppe an, so auch die endliche Bewegung, welche aus ihr durch unendlichmalige Wiederholung entsteht. DaB dies bei der Bewegung

*) Nor von diesen mechanischen Entwicklungen des Ballschen Werkes ist im vorliegenden Artikel die Rede, nicht von den anschließenden geometrischen. Ich möchte aber nicht unterlassen anzuführen, daß Herr Ball die geometrischen Fragen neuerdings in einer besonderen Abhandlung in den Transactions der R. Irish Academy (vol. 31, post 12, Dublin 1901) weiter verfolgt hat; dieselbe trägt den Titel: Further developments of the geometrical theory of six screws. 
starrer Körper keineswegs immer der Fall ist, zeigt das einfache Beispiel eines auf einer Ebene rollenden Zylinders. Hier treten daher die in $\$ 5$ genannten Gruppen von Bewegungen (bez. die mit ihnen verknüpften linearen Schraubensysteme von ,selbständiger gruppentheoretischer Bedeutung“) in charakteristischer Weise in den Vordergrund. In der Tat läBt sich die Kinetik aller dieser Gruppen genau so in Ansatz bringen wie in \$ 7 die Kinetik der Drehungen um einen Punkt und in $\$ 9$ diejenige der freien Bewegungen (eines starren Körpers); man wird sagen können, daB in allen diesen Fällen die Methode der Eulerschen Gleichungen eine naturgemäBe Verallgemeinerung findet.*) Die Gesamtheit der Bewegungen, welche ein starrer Körper nach der Natur der ihm auferlegten Bedingungen gegebenenfalls ausführen kann, ist immer in einer kleinsten Gruppe von Bewegungen enthalten. Es dürfte sich empfehlen, die kinetischen Gleichungen für den Körper jeweils so aufzustellen, daß man diese Gruppe als Ausgangspunkt nimmt, also bei ihr "kinematische Gleichungen" und das Analogon der Eulerschen Gleichungen aufstellt.

Göttingen, den 3. September 1901.

\section{Nachträgliche Bemerkungen.}

Den vorstehenden Artikel, der die Bedeutung der Ballschen Schranbentheorie für das Gesamtgebiet der Mechanik zusammenbängend darlegen und zugleich begrenzen soll, habe ich s. Z. verfaBt, weil es mir bei der Redaktion des Bandes IV der mathematischen Encyklopädie (der die Mechanik behandelt) erwünscht war, eine derartige Darstellung zur Hand zu haben; ich verweise in dieser Hinsicht auf den Artikel IV, 2 (Timerding, geometrische Grundlegung der Mechanik eines starren Körpers) und IV, 6 (Stäckel-Petersen, elementare Dynamik; erscheint demnächst). Wenn ich jetzt diesen Artikel in den Math. Annalen wieder abdrucke, so geschieht es, weil das Klassifikationsprinzip des § 1, dem ich allgemeine Bedeutung beilege, mit den sich daran anschliebenden Einzelausführungen seither nicht so beachtet scheint, wie ich es für richtig halte.

Vielleicht darf ich über die historische Entstehung dieses Prinzips hier folgendes bemerken. Der Gedanke, alle vorkommenden Größen nach ihrem Verhalten bei beliebigen linearen Transformationen zu klassifizieren, durchzieht bekanntlich die ganze Invariantentheorie und liegt bereits den ersten invariantentheoretischen Arbeiten von Cayley und Sylvester zugrunde. In meinem Erlanger Programm (1872) wurde sodann der Gesichtspunkt aufgestellt, daB die Gesamtheit der linearen Transformationen nur ein Beispiel irgend einer anderen Gruppe von Transformationen ist, denen man die jeweiligen Urvariabelen unterworfen denken mag. In Physik und Mechanik

*) Diese Bemerkungen stehen in naher Beziehung zu gewissen allgemeineren Betrachtungen über dynamische Probleme, die Herr Volterra in den Jahren 1899 bis 1900 in den Atti di Torino veröffentlichte; siehe insbesondere den Aufsatz: Sopra una classe di equazioni dinamiche in $\mathrm{Bd} . \mathbf{8 3}$ und den anderen: Sopra una classe di moti permanenti stabili in Bd. 34. 
hat man allon Anlaß, als solche Grappe eben die Hauptgruppe der räumlichen Änderungen, d. h. den Inbegriff der Bewegungen des Raumes und seiner Ähnlichkeitstransformationen zu wählen, und es ergibt sich dann durch sinngemäBe C̈bertragung der Anffassungsweise der Invariantentheoretiker das Klassifikationsprinzip des \& 1 mit Notwendigkeit. Ich habe dasselbe dementsprechend seit Jahren in meinen Vorlesungen zur Geltung gebracht, worauf auch Hr. Abraham in dem Encyklopädieartikel IV, 14 (Geometrische Grundbegriffe für die Mechanik der deformierbaren Körper), wo er das in Rede stehende Klassifikationsprinzip dnrchweg anwendet, ausdrücklich Berug nimmt.

Im ïbrigen ergibt sich, wie ich ausdrücklich hervorheber möchte, eben nach den Grundsätzen meines Nrlanger Programms, für die Darlegung und die Durchführung des Prinzips eine gewisse Latitüde. Cm dies nur nach einer Seite auszuführen: die "Hauptgruppe“ der räumlichen Änderungen ist eine Lntergruppe in der Gesamtheit der affinen Transformationen. Man kann unsere Klassifkation also in der Weise durchführen, dab man zunächst ein Schema der affinen Klassifikation aufstellt und in dieses dann die feineren Einzelheiten der metrischen Klassifikation erst hinterher einordnet. Eine wissenschaftliche Notwendigkeit, so vorzugehen, besteht aber keineswegs. Ich hebe dies hervor, um zu der Meinungsverschiedenheit Stellung zu nehmen, welche bei den neueren Diskussionen über die Grundlagen der Vektorenrechnung zwischen den Berren Mehmke und Prandtl hervorgetreten ist (Jahresbericht der Deutschen Mathematiker-Vereinigung, Bd. XIII, 1903),

Ich zitiere zum ScinluB gern noch einige neuerdings ersehienene Literatur, die zu den vorstehend wiederabgedruckten Entwickelungen in näherer Beziehung steht.

Zunächst ein Lehrbuch der analytischen Geometrie, in welchem die Unterscheidung der projektiven, affinen and metrischen (oder, wie die dutoren sagen, äquiformen) Geometrie in dem hier in Betracht kommenden Sinne von vorneherein mit Konsequenz durchgeführt wird. Es ist dies das Lehrbuch von Heffter und Köhler (Leipzig, erster Teil 1905).

Sodann, was Untersuchnngen über Schranbentheorie angeht, vor allen Dingen die nun vollendete Geometrie der Dynamen von Study (Leipzig, 1903), die neben vielem anderen Neuen, was über den Bereich des vorstehend abgedruckten Aufsatzes hinausliegt, insbesondere eine völlig durchgeführte Diskusion der verschicdenen Arten der linearen Schraubensysteme enthält. Ferner die Untersuchungen von Grünwald in den Bänden 48, 49 und 52 der Zeitschrift für Mathematik und Physik (1901, 1902, 1905), deren Titel ich hier wenigstens anführen will:

1) Sir Robert Balls lineare Schraubengebiete,

2) Zur Veranschaulichung des Schraubenbündels,

3) Darstellung aller Elementarbewegungen eines starren Körpers von beliebigem Freiheitsgrad.

Endlich, was die am Schlnsse meines Aufsatzes benutzte Untersuchung holonomer and nichtholonomer Geschwindigkeitskoordinaten angeht, die nenesten Publikationen:

Hamel, Die Lagrange-Eulerschen Gleichungen der Mechanik (in Bd. 5o der Zeitschrift für Mathematik und Physik, 1903), und Appell, Traité de Mécanique rationnelle, 2. Band, 2. Auflage (Paris 1904).

Göttingen, im Mai 1906. 